\title{
Efectos que tiene la Estimulación Temprana con un enfoque dirigido en la Memoria de Trabajo en niños de 4 años
}

\author{
Effects of Early Stimulation with directed approach in Working Memory in \\ children of 4 years old \\ Alexia Hernández-Luna ${ }^{a}$, Daniel Álvarez-Núñez ${ }^{b}$
}

\begin{abstract}
:
Early stimulation is defined as a set of repetitive, continuous and systematized actions through play that provides the child with the experiences he needs to fully develop his cognitive, linguistic, motor and social potential. Specific places such as the Early Stimulation Centers are intended to help children in a pleasant and fun way the development guidelines according to their age, using as a tool the directed and supervised game. The present work aimed to compare the effects of early stimulation with a targeted approach, in working memory in children of 4 years who were stimulated and in children who were not. The Neuropsychological Battery for Preschool (BANPE) test was used to assess the working memory visuospatial and verbal. The statistical procedure used was t-Student for independent samples finding homogeneity. Results: it was revealed that the experimental group has a higher score than the control group [ $\mathrm{t}(28)=5.80, \mathrm{p}<0.001]$, finding significant differences.
\end{abstract}

Keywords:

Working memory, early stimulationt

Resumen:

La estimulación temprana es definida como un conjunto de acciones repetitivas, continuas y sistematizadas a través del juego que proporciona al niño las experiencias que éste necesita para desarrollar al máximo sus potencialidades cognitivas, lingüísticas, motrices y sociales. Lugares específicos como los Centros de estimulación temprana tienen como finalidad ayudar a los niños de una manera placentera y divertida los lineamientos de desarrollo acorde a su edad, utilizando como herramienta el juego dirigido y supervisado. El presente trabajo tiene como objetivo comparar los efectos que tiene la estimulación temprana con un enfoque dirigido, en la memoria de trabajo en niños de 4 años que fueron estimulados y en niños que no lo fueron. Se utilizó como prueba la Batería Neuropsicológica para Preescolar (BANPE) para evaluar la memoria de trabajo visoespacial y verbal. El procedimiento estadístico utilizado fue t-Student para muestras independientes encontrando homogeneidad. Resultados: se reveló que el grupo experimental tuvo una puntuación mayor a la del grupo control [ $\mathrm{t}(28)=5.80, \mathrm{p}<0.001]$, Los resultados de este estudio validan las investigaciones que han señalado los beneficios de la ET a largo plazo en diversas áreas del desarrollo del niño.

Palabras Clave:

Memoria de Trabajo, Estimulación Temprana

\section{Introducción}

Los cambios relacionados al desarrollo del cerebro se deben a la interacción entre la biología y el ambiente en que se desenvuelve el niño. Tales cambios no están definidos solamente por la genética, sino por los efectos de una estimulación adecuada dentro del hogar o de una institución. La falta de intervención temprana en los niños puede llegar a provocar un retraso en las habilidades y destrezas en el desarrollo cognitivo (Bolaños, 2005). Por lo tanto, la intervención en los primeros años de vida es determinante para el desarrollo

\footnotetext{
Centro de Enseñanza Técnica y Superior, Universidad Campus Ensenada, https://orcid.org/0000-0002-4955-7323, Email: hlunaalexia@gmail.com

b Centro de Enseñanza Técnica y Superior, Universidad Campus Mexicali, https://orcid.org/0000-0003-2883-9572, Email: daniel.alvarez@cetys.mx
} 
del ser humano (Baker y López, 2013; Ostrosky et al., 2016).

La estimulación temprana (ET) es definida como un conjunto de acciones repetitivas, continuas y sistematizadas a través del juego que proporciona al niño las experiencias que éste necesita para desarrollar al máximo sus potencialidades cognitivas, lingüísticas, motrices y sociales (Vázquez et al., 2018). Lugares específicos como los Centros de ET, tienen como finalidad ayudar a los niños de una manera placentera y divertida los lineamientos de desarrollo acorde a su edad, utilizando como herramienta el juego dirigido y supervisado. De igual manera, motiva a los papás a formar parte de su enseñanza, interactuando con ellos durante la aplicación de los ejercicios (Garza, 2014).

A pesar de la existencia de investigaciones acerca de los beneficios de la ET a largo plazo en diversas áreas del desarrollo, la mayoría de las investigaciones dejan a un lado los aspectos cognitivos dirigidos a procesos específicos (Zubia et al., 2016; Edmond et al., 2019; López, 2013). Un proceso cognitivo que se ha mostrado relevante en cuanto a los beneficios en el ámbito escolar es la memoria de trabajo (MT), debido a su capacidad para almacenar y procesar información durante periodos cortos de tiempo, siendo un proceso cognitivo primordial para el aprendizaje escolar (Harmony, 2004). Asimismo, la MT es considerada esencial para funciones cognitivas superiores tales como, el razonamiento, funciones ejecutivas, planificación y la comprensión lectora (Bull et al., 2008; Schneider,2014). Por lo tanto, dicho proceso cognitivo podría ser un predictor del éxito académico a largo plazo, específicamente en las habilidades matemáticas y lectoras.

\section{Método}

La población del estudio se llevó a cabo con participantes de cuatro años de edad, los cuales cursaban 1 ro de preescolar. El número total de la muestra fue de 30 niños, de los cuales 15 pertenecían al grupo que acudieron a un Centro de ET y los otros 15 niños no habían asistido a un Centro de ET.

\section{Criterios de inclusión:}

Candidato 1:

a. Niños en primero de preescolar.

b. Edad de cuatro años.

c. Padres con al menos escolaridad media básica completada.

d. Haber acudido desde los 6 meses de nacidos hasta el 1. 4 meses a un Centro ET con un enfoque dirigido. Contando con un programa enfocado al desarrollo integral del niño: Estimulación visual, auditiva, sensorial, cognitiva, motora y social. En sesiones de 45 minutos una vez por semana.

Candidato 2:

e. Niños en primero de preescolar.

f. Edad de cuatro años.

g. Padres con al menos escolaridad media básica completada.

h. No haberacudido desde los 6 meses de nacidos a un Centro de ET con un enfoque dirigido.

\section{Criterios de no inclusión}

a. Niños menores o mayores de cuatro años.

b. Niños no escolarizados.

c. Padres sin educación media básica completada.

d. Trastornos del Desarrollo Neurológico DSM-5.

e. Factores de riesgo:

- Prenatales: desnutrición, alcoholismo, drogadicciones, medicamentos (corticoesteroides, diacepam, cloropromacina, fonobarbital, anticonvulsivantes), contaminantes químicos, enfermedades infecciosas (rubeola, varicela, toxoplasmosis, citomegalovirus) (Zubia et al., 2016).

- Perinatales: asfixia perinatal, bajo peso al nacer, prematurez (Zubia et al., 2016).

- Posnatales: infecciones del sistema nervioso, desventaja sociocultural, restricción ambiental, desnutrición, trau mas craneales, enfermedades cardiacas, fallas de audición, fumar por parte de la madre en la lactancia, quimioterapia, inhalación de solventes (Zubia et al., 2016).

\section{Materiales e instrumentos}

El instrumento que se utilizó en el estudio fue la Batería Neuropsicológica para Preescolar (BANPE). A continuación, se describe cada una de las tareas y técnicas utilizadas para la recolección de datos:

\section{Memoria de trabajo verbal}

- Dígitos en regresión: La tarea consiste en que el examinador lea en voz alta una serie de números, la cual el niño deberá repetir en orden inverso. La longitud de la serie va aumentando progresivamente, hasta que el niño falle en dos ocasiones consecutivas en el mismo nivel. El puntaje obtenido es equivalente al número máximo de dígitos que el sujeto logre repetir en esta prueba. 
Memoria de trabajo visoespacial

- Cubos en regresión: La resolución de esta tarea consiste en que el evaluador señale una serie de cubos a un ritmo de 1 cubo por segundo. Posteriormente, se le pide al niño que señale los cubos en orden inverso. La longitud de la secuencia incrementa hasta que el niño cometa dos fallos seguidos en el mismo nivel. El puntaje obtenido es equivalente al número máximo de cubosque el sujeto logre señalaren esta prueba.

- Vaca que reparte leche:La tarea consiste en que la vaca irá repartiendo tarros de leche a unos personajes que ya están ordenados espacialmente en una lámina, el número de los personajes a los que dejará la leche irá incrementando conforme se avance en la tarea. El objetivo es que el niño recoja los tarros de leche de forma inversa a como la vaca los dejó en un inicio. El puntaje obtenido es equivalente al número máximo de tarros de leche que el sujeto logre recoger en esta prueba de forma inversa.

\section{Procedimiento}

La primera etapa del procedimiento fue el acercamiento a las dos instituciones donde se encontrarán las muestras seleccionadas para el estudio. Para obtener la muestra de los niños que recibieron ET, se acudió a un preescolar privado, y para obtener la muestra de los niños que no recibieron ET, se atendió a un jardín de niños. Después, se revisaron en ambas instituciones expedientes escolares de los posibles candidatos, con el objetivo de seleccionar los participantes que cumplieran con los criterios de inclusión y no inclusión. Posteriormente, se establecieron fechas para acudir a la hora de salida de los niños de ambos preescolares con el fin de platicar personalmente con cada padre de familia solicitando su autorización, y a la vez, hacerles entrega de los consentimientos informados. Finalmente, se asignaron fechas en ambas instituciones para llevara cabo las tres tareas de la BANPE alos niños. Se consideraron aproximadamente de 15 minutos por aplicación.

\section{Análisis Estadístico}

Para contrastar las hipótesis propuestas en la presente investigación, se realizó un análisis paramétrico por medio de la prueba $t$ de Student para grupos independientes. Para llevar a cabo el análisis, se hizo uso del programa estadístico IBM Statistical Package for the Social Sciences (SPSS) versión 24.

\section{Resultados}

Después de aplicarse las tareas de memoria de trabajo de la prueba BANPE, se encontró que el grupo control (GC) tuvo una media de 95.60 y una desviación estándar de 10.99; y el grupo experimental (GE), obtuvo una media de 116.60 y una desviación estándar de 8.69. (ver tabla 1).

\section{Tabla 1.}

Estadísticos descriptivos de los resultados obtenidos de las tareas de MT de la prueba BANPE en el GE y GC.

\begin{tabular}{|c|c|c|c|c|}
\hline \multicolumn{2}{|c|}{ GRUPOS } & $N$ & Media & Desv. \\
\hline \multirow[t]{2}{*}{$M T$} & GE & 15 & 116.60 & 8.69 \\
\hline & GC & 15 & 95.60 & 10.99 \\
\hline
\end{tabular}

El análisis estadístico realizado mediante la prueba t de Student para muestras independientes reveló que el GE tuvo una puntuación mayor a la del grupo control [t $(28)=5.80, p<0.001]$ (ver figura 1).

\section{Figura 1.}

Análisis estadísticos de la prueba $t$ de Student de la evaluación de MT de la prueba BANPE.

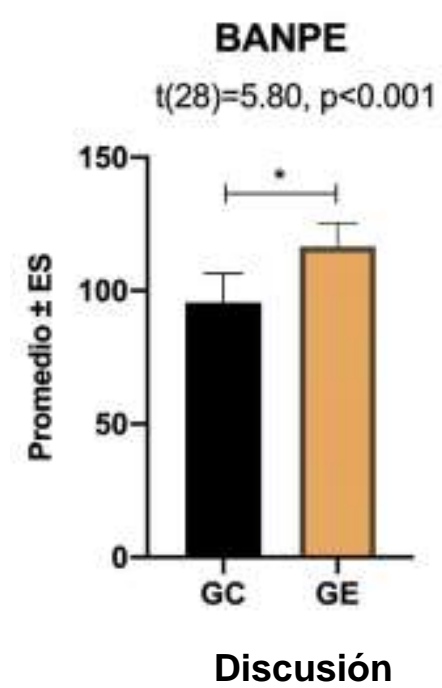

En estudios se ha señalado que la ET permite potencializar el desarrollo cognitivo, lingüístico, motriz y social del niño (Vázquez et al., 2018). Sin embargo, la mayoría de las investigaciones dejan a un lado los aspectos cognitivos dirigidos a procesos específicos. Por lo tanto, se pretendió investigar si los niños que fueron estimulados a temprana edad, y que actualmente tienen una edad de 4 años, mostraban mayor desempeño en la 
MT. Se encontró que los niños que habían recibido ET desde los 6 meses hasta el 1.4 meses desempeñaron una clasificación normal alta en la MT, mientras los niños que no recibieron ET contaron con una clasificación normal en la MT. Tales resultados validan las investigaciones que han señalado los beneficios de la ET a largo plazo en diversas áreas del desarrollo del niño (Zubia et al., 2016; Edmond et al., 2019; López, 2013). No obstante, los niños que no recibieron ET no contaron con alguna alteración en la MT, obteniendo una clasificación dentro de la norma (ver figura 1). Lo cual indica que el nivel edu cativo de los padres, la estimulación en el ámbito familiar, los cuidados y habilidades lingüísticas parentales tienen un impacto en el desarrollo de las competencias cognitivas de los niños como se ha reportado en otros estudios (Hauser, 2010).

\section{Conclusiones}

Los resultados de este trabajo generaron dos conclusiones relevantes; la ET que recibieron los niños potencializó la MT, logrando especificar un proceso cognitivo que se muestra beneficiado por la metodología impartida en la ET con un enfoque dirigido. Y la ausencia de la asistencia a un Centro de ET nogenera desventajas significativas en el rendimiento de la MT; dado que los niños que no recibieron ET contaron con un rendimiento normal.

La investigación logró identificar un proceso cognitivo que se logra potencializar por medio de laET con un enfoque dirigido. Sin embargo, se recomienda para futuras investigaciones profundizar en aquellos niños que recibieron ET con un enfoque dirigido y el efecto en sus habilidades matemáticas y lectoras en el ámbito escolar. Asimismo, es importante continuar identificando cuáles procesos cognitivos se muestran beneficiados por la ET profundizando en el análisis de la metodología impartida en los Centros de ET.

\section{Referencias}

Baker-Henningham, H., \& López Bóo, F. (2013). Intervenciones de estimulación infantil temprana en los países en vías de desarrollo: Lo que funciona, por qué y para quién. https://publications.iadb.org/publications/spanish/docume nt/Intervenciones-de-estimulaci\%C3\%B3n-infantiltemprana-en-los-pa\%C3\%ADses-en-v\%C3\%ADas-dedesarrollo-Lo-que-funciona-por-qu\%C3\%A9-y-paraqui\%.C3\%A9n.pdf

Bolaños, M. (2005). Aprendiendo a estimular al niño: Manual para padres y educadores con enfoque humanista. Recuperado https://books.google.com.mx/books?id=P2uFhtS68VoC\& printsec $=$ frontcover\&source $=g b s \_g e \_s u m m a r y \_r \& h l=e s \#$ $\mathrm{v}=$ onepage $\& \mathrm{q} \& \mathrm{f}=$ false

Bull, R., Espy, K. A., \& Wiebe, S. A. (2008). Short-term memory, working memory, and executive functioning in preschoolers: Longitudinal predictors of mathematical achievement at age 7 years. Developmental neuropsychology, 33(3), 205-228.
Edmond, K. M., Strobel, N. A., Adams, C., \& McAullay, D. (2019). Effect of early childhood development interventions implemented by primary care providers commencing in the neonatal period to improve cognitive outcomes in children aged 0-23 months: protocol for a systematic review and meta-analysis. Systematic reviews, 8(1), 224.

Garza, Jessica. (2014) El impacto de la Estimulación Temprana en la primera infancia. Tesis de Licenciatura. Universidad de Monterrey.

Harmony, T. (2004). Factores que inciden en el desarrollo del sistema nervioso del niño. In: $M$. Corsi Cabrera, ed., Aproximaciones de las neurociencias a la conducta., 2nd ed. Universidad de Guadalajara: El Manual Moderno, 147-158.

Hauser, P., y Labin, A. (2018). Evaluación cognitiva de niños: un estudio comparativo en San Luis, Argentina/Cognitive Evaluation of Children: a Comparative Study in San Luis, Argentina. Revista Costarricense de Psicología, 37(1), 27-40.

López, M. (2013). Rendimiento académico: su relación con la memoria de trabajo. Revista Electrónica "Actualidades Investigativas en Educación (pp. 3-7).

Ostrosky, F. (2010). Desarrollo del cerebro. Neurociencias, Universidad Nacional Autónoma, (pp.1-10).

Ostrosky, F., Lozano, A. y González, M. (2016). Batería neuropsicológica para preescolares (BANPE). https://www.pearsonclinical.es/banpe-baterianeuropsicologica-para-preescolares

Schneider, W. (2014). Memory development from early childhood through emerging adulthood. Springer, 188-189.

Vázquez, B. C., Ramos, M. B., \& Córdova, A. E. (2018). El juego en la estimulación temprana del desarrollo en niños preescolares. Revista Electrónica de Psicología de la FES Zaragoza, UNAM. Vol. 8, No. 15, enero-junio, 2018, (pp. 18-25).

Zubía, R. F. (2016). Efectos de un programa de estimulación temprana en el desarrollo integral de niños de 1 a 12 meses. Revista Mexicana de Investigación en Cultura Física y Deporte, 1(1), 174-187. 